\title{
Morphodynamic modelling for the entire German Bight: an initial study on model sensitivity and uncertainty
}

\author{
A. Plüß and F. Kösters \\ Federal Waterways Engineering and Research Institute, Hamburg, Germany \\ Correspondence to: A. Plüß (andreas.pluess@baw.de) \\ Received: 15 October 2013 - Revised: 15 January 2014 - Accepted: 17 January 2014 - Published: 1 April 2014
}

\begin{abstract}
Morphodynamic modelling of coastal seas and estuaries for large-scale and long-term applications is strongly affected by parameter sensitivity of process-based models. Moreover, the comparison of data-based methods with numerical model results is limited by uncertainties in measurements. These drawbacks can be partly overcome by a multimodel approach (MMA). In a case study to assess longterm sediment transport and morphodynamic processes for the German Bight, the AufMod research project applies two different methods for process-based modelling: UnTRIMSediMorph and DELFT3D. Model sensitivity is illustrated in terms of different morphological changes for diverse porosity values. As a first step, discrepancies between individual methods are shown based on resulting sediment transport patterns.
\end{abstract}

\section{Introduction}

The North Sea acts as a gateway for container ships connecting European harbours to the world. Within this economical important region, natural and man-made morphological changes take place which potentially affect safety and ease of maritime traffic. The prediction of morphologic changes on temporal and spatial scales is relevant for coastal engineering applications and although it is still a scientific challenge (e.g. French and Burningham, 2009). The application and interpretation of (long-term) morphodynamic model results demand for well-founded knowledge about sediment transport and local morphological conditions in the field as well as profound knowledge of the modelling techniques.

In order to improve our current understanding of these morphological changes, the multidisciplinary research project AufMod (German acronym for "Model-based analy- sis of long-term morphodynamic processes in the German Bight") was funded by the Federal Ministry of Education and Research (BMBF) to investigate long-term sediment transport and morphodynamic processes. The project focuses on the German Bight, located in the south-eastern part of the North Sea. AufMod takes a combined data-based and process-based modelling approach to investigate long-term sediment transport. One main scientific objective of AufMod is to identify processes and effects which are relevant for the long-term sediment transport and the morphodynamic reaction of the seabed. The first step is to investigate the uncertainty of the simulation results from different numerical methods and parameterizations.

In order to simulate sediment transport, a consistent database for bathymetric and sedimentological data is indispensable. Only when these data are available, hydrodynamic transport models can be utilized to calculate largescale sediment transport; as a consequence of this the morphological changes. In practice there is often not sufficient field data (e.g. bathymetry, sediment properties) available to calibrate and validate a morphodynamic (MD) model thoroughly enough for a reliable forecast. The calibration is further complicated by uncertainties inherent to the numerical methods (e.g. sediment transport formulations, numerical diffusion) and physical processes not or not well represented (e.g. erodibility or stability of sediments at the bed, consolidation).

In this situation, the MD multi-model approach (MMA) offers a solution to overcome the uncertainties mentioned above for sediment transport and morphodynamic studies (Plüß and Heyer, 2008). Similar to the approach in climate models, covering the range of potential changes in the climate as a response to an increase in $\mathrm{CO}_{2}$ (e.g. Tebaldi and Knutti, 2007), the MMA is in particular helpful to determine 
applicability, skill and uncertainty of available modelling systems in sediment transport studies.

In this study we focus on two aspects of sensitivity and uncertainty of numerical model results. First, bed level changes calculated from MD models heavily depend on the sedimentological properties prescribed as boundary conditions for the bed. Therefore, necessity and reliability of sedimentological properties need to be assessed. If it is possible to calculate the distribution and sorting of the seabed sediment, one might be able to reduce the amount of input data for morphodynamic model simulations. Moreover, what is the impact of observed variability in key sedimentological properties such as the initial sediment grain size distribution or the porosity of surface sediments? The initial sediment distribution is generally not well known and will vary on scales smaller than those resolved by the model. Porosity depends on the sorting of sediments as well as on compaction and thus the sedimentological history, which is not commonly taken into account in present-day morphodynamic models. The second aspect, covered in this study, is to compare sediment transport patterns from different modelling systems and thus the parameter sensitivity of different empirical sediment transport formulations.

\section{Methods}

Two different modelling systems are employed here. Firstly, the unstructured 3-D hydrodynamic model UnTRIM (Casulli and Zanolli, 2002) coupled with the sediment transport model SediMorph (Malcherek et al., 2005) and the unstructured version of the wave model K-model (Schneggenburger, 1998) was applied. Secondly, the structured model DELFT3D (Stelling, 1984, 1986) was used in a 2-D mode, combining hydro-, sediment and morphodynamics (Lesser et al., 2004) together with the effects of waves (Booij and Holthuisen, 1996).

The MD simulations cover the time span from 1996 to 2008 and are steered using spatial and temporal varying forcing based on operational forecast model results provided by national authorities, taking into account the whole variability of tides, external surge, river run-off, wind and waves. The large-scale and long-term sediment transport models comprise coastal areas, islands, shore lines and connected estuaries. Because of the versatile effects and interactions between the individual regions (estuary-coastal region-shelf), different approaches for grid generation of the individual methods were necessary. The unstructured UnTRIM model consists out of 77500 triangular elements with a resolution varying from $24 \mathrm{~km}$ in the outer North Sea down to $80 \mathrm{~m}$ in the estuaries (BAW, 2013). The vertical resolution changes from $50 \mathrm{~m}$ off the coast down to $1 \mathrm{~m}$ in intertidal areas. The structured DELFT3D model was set up in a vertical integrated (2-D), nested configuration: (a) North Sea model with $455 \times 489$ curvilinear grid cells with a variable resolution from $1 \mathrm{~km}$ in the outer North Sea down to $1 \mathrm{~km}$ near the coast and (b) German Bight model with $762 \times 1046$ curvilinear grid cells with a variable resolution from $1.8 \mathrm{~km}$ in deep water down to $100 \mathrm{~m}$ in the estuaries.

At the open boundaries of both North Sea models, water levels predicated on tidal constituents out of the global model FES 2004 (Lyard et al., 2006) and external surges based on the operational model of the German Hydrographic Office (BSH) have been prescribed. UnTRIM solves the hydrodynamics using a time step of $120 \mathrm{~s}$; DELFT3D uses $150 \mathrm{~s}$. River run-off is taken to be variable within the inner German Bight resulting out of measurements. For the continental run-off, seasonally averaged mean values were assigned. The atmospheric forcing (wind speed) is taken from model results of the operational model of the German National Meteorological Service (DWD). The wave model has been subsequently run with pre-calculated water levels and the same atmospheric forcing as the hydrodynamic model.

For the long-time calculations annual bathymetries are computed with the utilization of the time varying digital bathymetry model year by year provided by the AufMod database (see http://projekt.mdi-de.org/services/verwandteprojekte/40-aufbau-von-integrierten-modellsystemen.html).

For long-term morphodynamic simulations, it is crucial to prescribe the sediment distribution and composition of the seabed precisely. For this, the initial sediment distribution and composition are taken from the functional AufMod sediment model, which integrates more than 76500 measurements of grain size distribution.

Sediment transport in UnTRIM is modelled as transport of individual sediment classes either transported as suspension or as bed load following the formulation of van Rijn (1993). In the DELFT3D model the standard sediment transport formula (van Rijn 1993) is chosen taking into account both suspended and bed-load transport.

The hydrodynamic numerical $(\mathrm{HN})$ model results have been validated using more than 90 tide gauge stations around the North Sea yielding sufficient accuracy of modelled water levels (BAW, 2013). Also, the simulated waves show good agreement to measurements. For suspended sediment and bed load transport, no detailed validation could be carried out as measurements appear too seldom and are only locally available. Moreover, the validation of the MD modelling results has been inhibited due to only sparse measurements in time and space, with accuracies from the measurements of the order of the morphological changes within the simulation period.

As an alternative calibration approach, the local bed shear stress, based on hindcast simulations, has been shown to correlate with the measured mean bed evolution range significantly (BER denotes the difference between the lowest and highest seabed elevation in the time span considered; Kösters and Winter, 2014). 


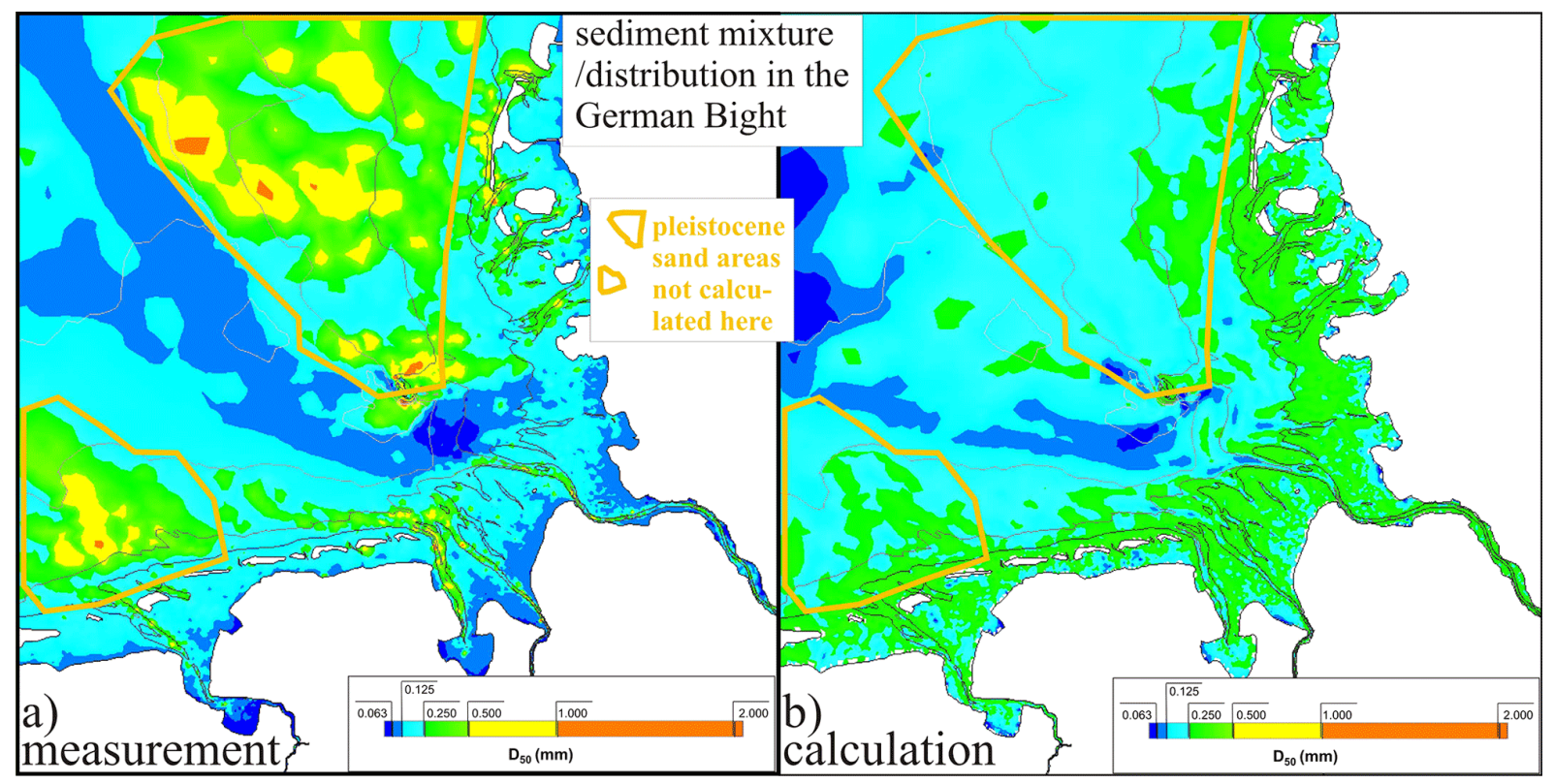

Fig. 1. Initial sediment mixture/distribution in the German Bight from (a) measurements and (b) numerical model calculation (UnTRIM).

\section{Results and discussion}

\subsection{Reliability of sedimentological properties as model input data}

To address the question if sedimentological properties of the bed can be obtained from a hydraulic sorting of sediments in order to avoid expensive measurements, a model study has been conducted. An UnTRIM simulation run is started with an initially uniform sediment distribution and compared to the measured distribution. The sediment distribution of the German Bight (Fig. 1a) was obtained as median grain size (D50) from the AufMod database. This data set updates and extends previous work (Figge, 1981) by including more than 7600 sediment samples from the German Bight. The derived D50 data have been mapped on the numerical model grid. The state of sediment distribution results from sorting of Holocene sediments due to hydrodynamic forcing and outcropping Pleistocene sediments in regions of Holocene sediment deficiency (Zeiler et al., 2008). Sediments mainly consist of well-sorted fine sand with regions of medium sand at the North Frisian coast. In the northern part of the East Frisian shelf, glacial gravel deposits can be found. Finer sediments are present in the Wadden Sea (e.g. at tidal flats, in the estuaries, in the mud deposition area close to Helgoland and in the drowned river Elbe valley). In tidal channels or in tidal inlets, coarser sediments are observed. The model calculates the sorting of bed material for a run time of 60 days taking into account the effects of tidal, wind- and wave-driven bed shear stresses. The initial sediment distribution has been set to a uniform distribution of spatially averaged conditions of the German Bight as obtained from the
AufMod data set represented by four bed load fractions of the following: very coarse sand (vcSa, grain size 1500 mum, fraction $0.3 \%$ ), coarse sand (cSa, 750 mum, $2.9 \%$ ), medium sand (mSa, 375 mum, 16.2\%), fine sand (fSa, 187.5 mum, $36.5 \%)$ and five suspended load fractions of very fine sand (vfSa, 94 mum, 25.9\%), coarse silt (cSi, 46.5 mum, 9.6\%), medium silt ( $\mathrm{mSi}, 23.5$ mum, $3.7 \%$ ), fine silt (fSi, 12 mum, $3.3 \%$ ), and very fine silt (vfSi, 6 mum, $1.6 \%$ ). The resulting sediment distribution from the sorting model experiment is shown as D50 in Fig. 1b.

Although an exact reproduction of the sedimentology of the German Bight cannot be expected from the numerical model, several characteristic features are reproduced. The comparison of the observed and modelled sediment distribution shows that typical accumulation areas of finer sediments behind barrier islands, in estuaries and in extension of the estuaries are reproduced by the model. Coarser sediments in tidal channels of estuaries and tidal inlets are mostly captured but tend to be underestimated in the model results. Deposits of coarse sand on the shelf can only partly be captured by the model; notably the extensive area of coarse sediments on the North Frisian shelf cannot be reproduced. Sediment sorting in energetic tidal channels mainly reflects present-day tidal forcing conditions and can qualitatively reproduce characteristic features after only 60 days of hydrodynamic forcing. Similarly the redistribution of fines in back-barrier regions becomes apparent after this short period. However, main features of the observed sediment properties are absent such as coarse sediments at the North Frisian shelf which originate from glacial periods. Moreover, even though the sediment distribution is qualitative similar, the grain size distribution can differ quantitatively by more than an order of magnitude. 


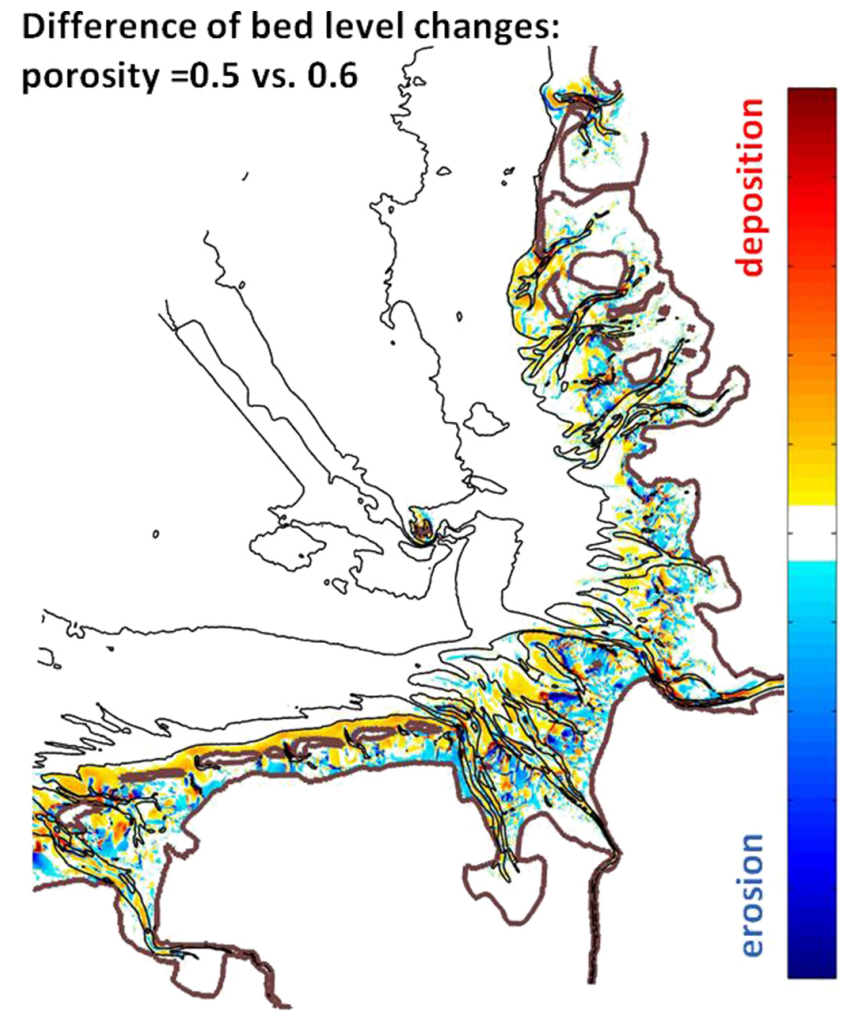

Fig. 2. Differences in bed level changes after $1 \mathrm{yr}$ MD simulation with DELFT3D (2-D mode) due to a change of the porosity parameter from 0.5 to 0.6 .

As the sediment transport strongly depends on the sediment grain size (as can be seen easily from sediment transport formulas, e.g. van Rijn 1993), it has to be concluded that, for study areas with inhomogeneous sediments such as the German Bight, hydrodynamically calculated sediment distributions are not reliable for large-scale sediment transport applications. This emphasizes the need for a detailed sedimentological investigation prior to numerical modelling. And it is not only the sediment grain size distribution which affects model results but also the space variable porosity of the seabed.

The porosity of surface sediments is not well known and difficult to measure. Due to this, commonly the assumption of a space and time constant porosity is made. It is expected that larger porosity yields a stronger morphological reaction because sediment movement is easier if less densely packed, and the same amount of transported sediment results in a larger volume change. In MD models such as DELFT3D, morphological changes are calculated from residual sediment mass transport and then are converted into volume changes using the user-defined porosity, and a strong influence is evident. As an example, the difference in bed evolution after $1 \mathrm{yr}$ of simulation for changing the porosity from 0.5 to 0.6 is assessed as a sensitivity study. The experiment has been carried out using the DELFT3D mod- elling system applying five sediment fractions: coarse sand (cSa, 750 mum), medium sand (mSa, 375 mum), fine sand (fSa, 187.5 mum), coarse silt (cSi, 46.5 mum) and fine silt (fSi, 12 mum) together with the default DELFT3D settings for sediment transport. The differences in bed level changes between the two porosity settings show a spatially structured response (Fig. 2). In deeper areas off the coast and islands, more deposition and less erosion occur. In contrast to this, shallow tidal areas are more erosive, and less deposition can be found here. The physical mechanism behind this is that with increased porosity sediments from the tidal flats can be more easily mobilized by, for example, wave action and then be transported by tidal currents to deeper areas.

Again, these results illustrate the importance of a precise description of the sediment properties at the seabed for reliable morphodynamic calculations.

\subsection{Uncertainties and differences of sediment transport for different modelling systems}

The two aforementioned modelling systems UnTRIMSediMorph and DELFT3D have been calibrated independently for the year 2006. Both models are compared in order to investigate the effect of the different numerical methods and model parameterizations.

To demonstrate the main differences and similarities in time and space, the spatial distribution of sediment transport is shown for bed load transport (Fig. 3) and suspended load transport (Fig. 4). In addition a time series at Helgoland is presented (Fig. 5). The spatial sediment transport is shown for a stormy situation on 9 February 2006 during flood (Figs. 3, 4 left) and ebb (Figs. 3, 4 right) tide.

In both model simulations the spatial distribution of bed load transport is more pronounced near the coast and the estuary mouths for both flood and ebb tide. In contrast, the suspended load transport is more widespread over the German Bight. During flood tide the maximum is located near the coast/estuary mouths; during ebb tide suspended sediment transport is extended seaward. Overall the areal distributions between both models show qualitatively a good agreement; quantitatively the results vary due to differences in model parameterizations. Note that DELFT3D is set up as a depthintegrated 2-D model, whereas UnTRIM-SediMorph is run in 3-D with a vertical resolution between $1 \mathrm{~m}$ and $50 \mathrm{~m}$.

The temporal distribution of sediment transport is shown in Fig. 5 as time series of depth-integrated sediment transport at Helgoland, which is located in the inner German Bight. For all three time series, the same axis dimension is used for a better comparison. The time span covers a complete springneap cycle (4-19 February 2006) and a storm situation (9 February 2006). Bed load transport quantities (top panel) are small compared to the suspended transport (middle panel). In the DELFT3D (2-D) run, the bed load is more dominant compared to UnTRIM (3-D). Note that the form of transport mode (suspended load, bed load) of individual sediment 

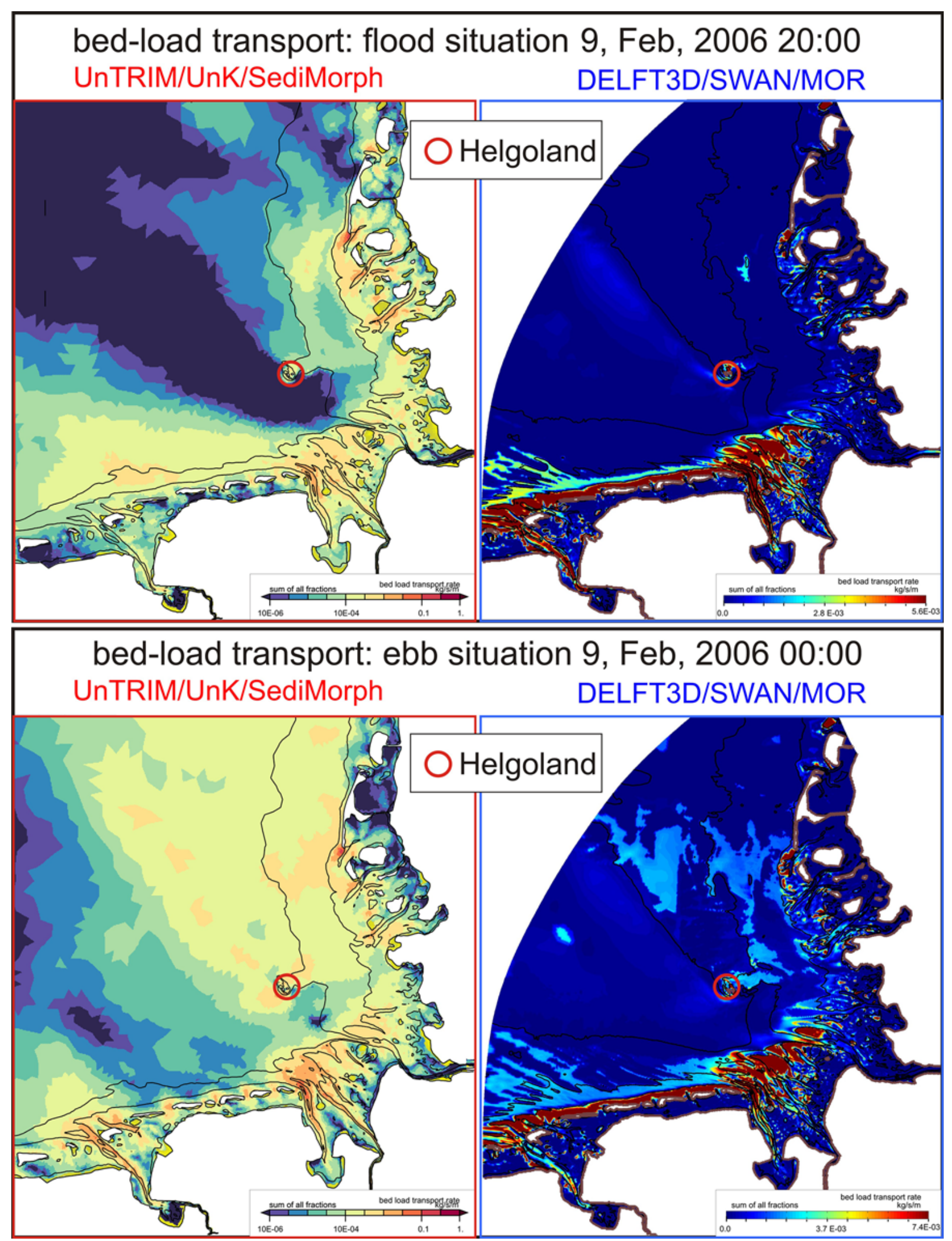

Fig. 3. Bed load transport for windy flood/ebb velocity tide in the German Bight, calculated by UnTRIM/UnK/SediMorph and DELFT3D(2D mode)/SWAN/MOR. 


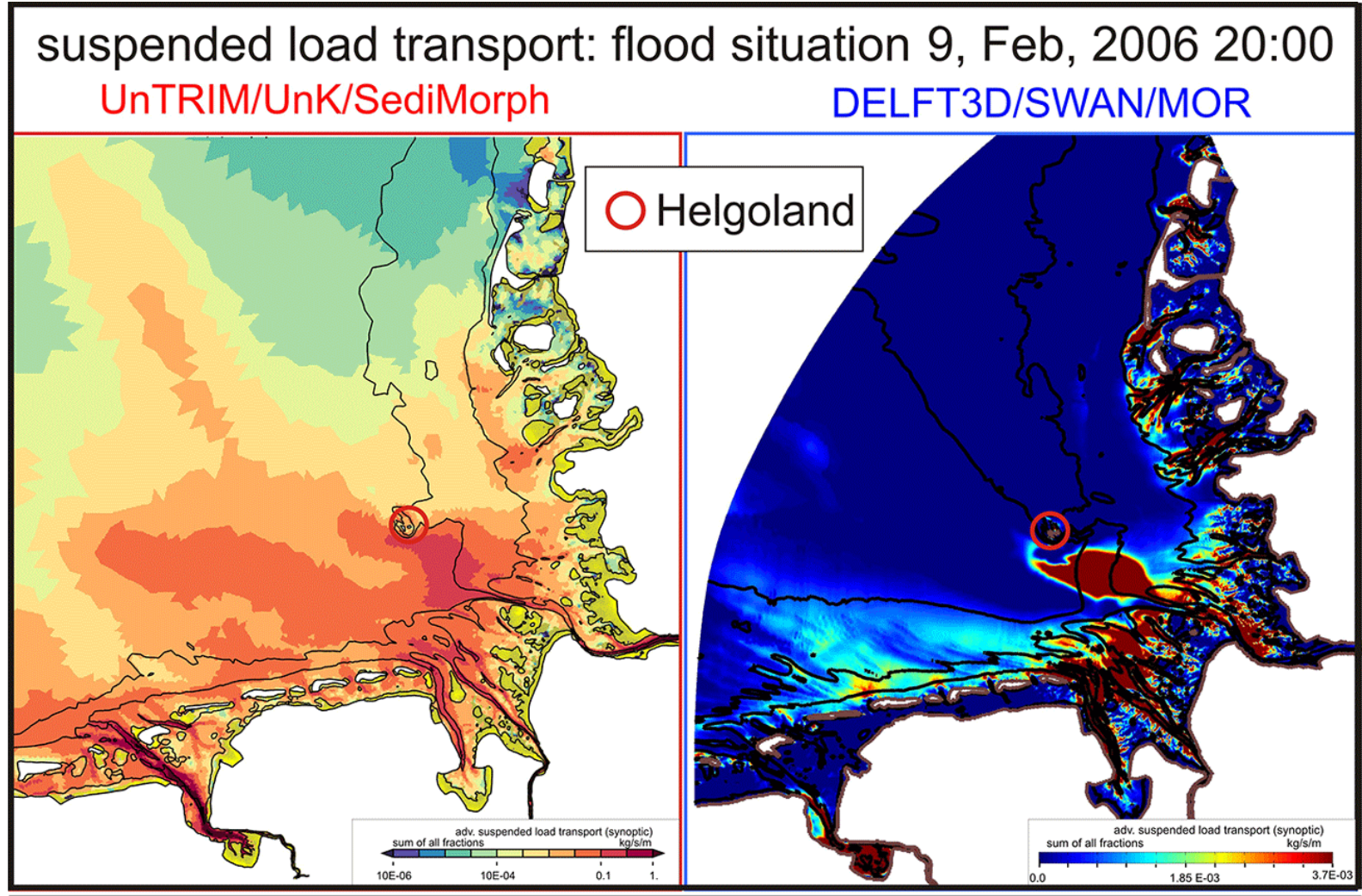

suspended-load transport: ebb situation 9, Feb, 2006 00:00 UnTRIM/UnK/SediMorph DELFT3D/SWAN/MOR

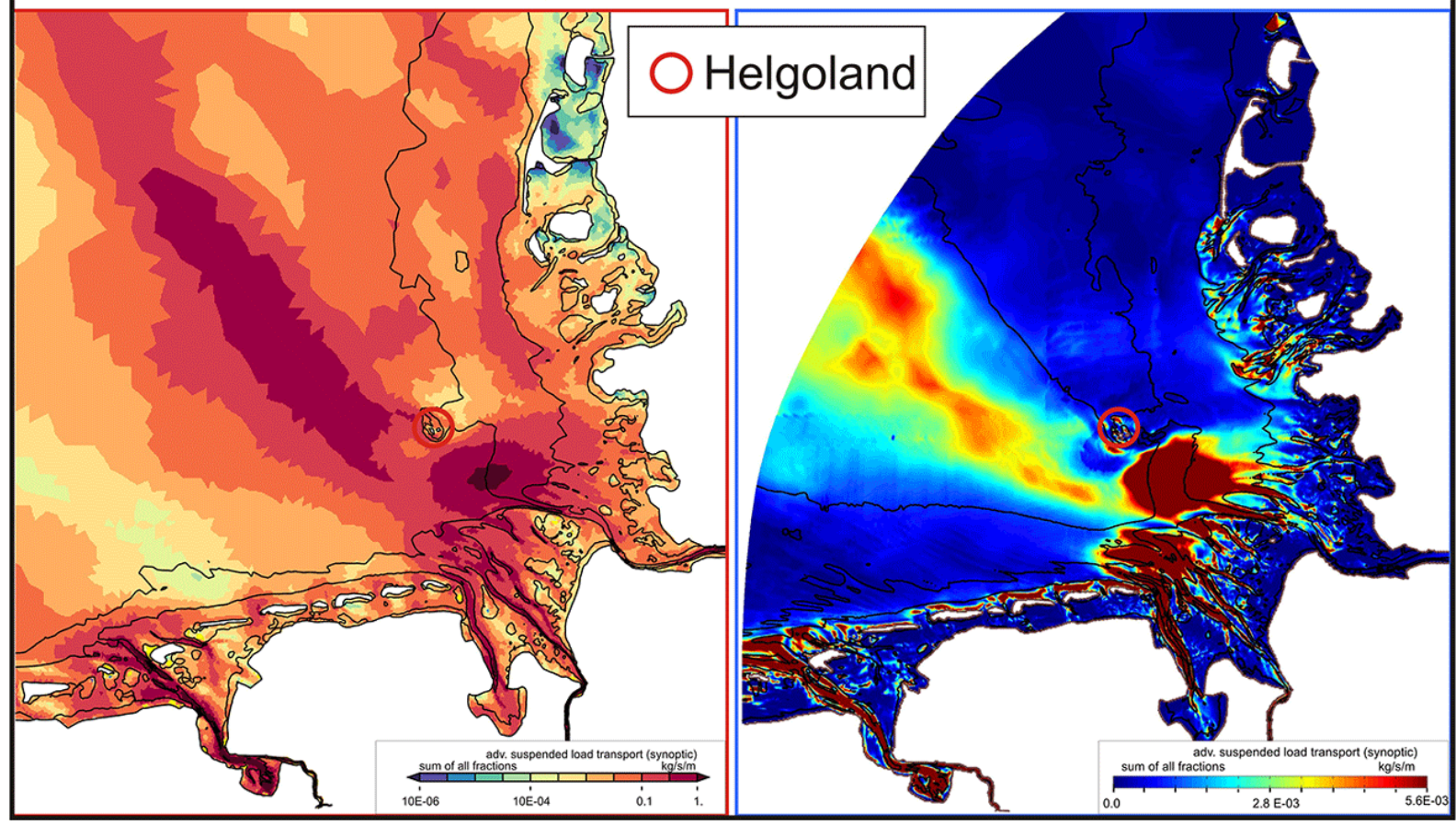

Fig. 4. Suspended load transport for windy flood/ebb velocity tide in the German Bight, calculated by UnTRIM/UnK/SediMorph and DELFT3D(2-D mode)/SWAN/MOR. 


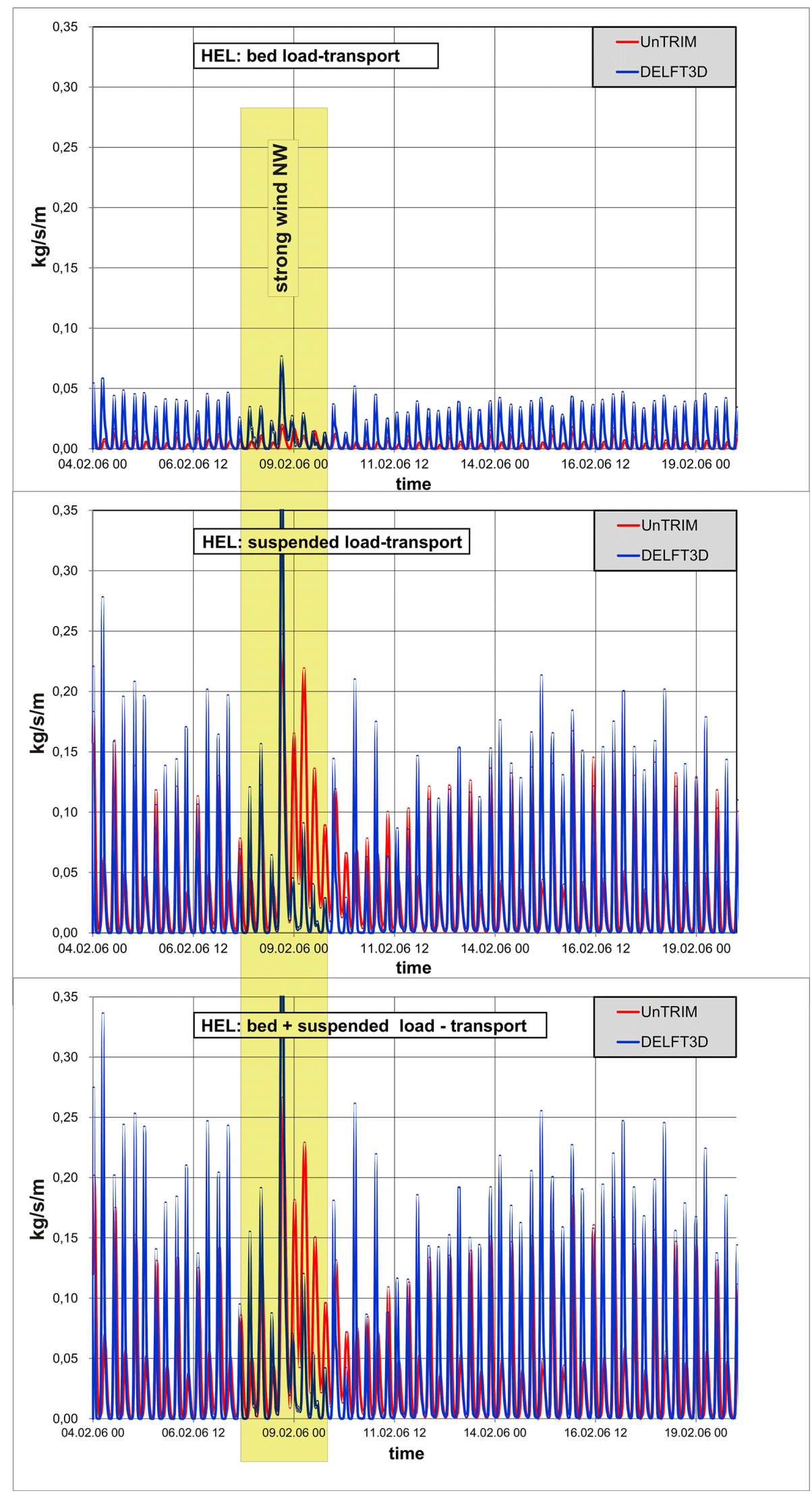

Fig. 5. Calculation of bed load, suspended load and total load transport at Helgoland (February 2006) derived from UnTRIM/UnK/SediMorph and DELFT3D(2-D mode)/SWAN/MOR. 
fractions is internally determined in DELFT3D based on the flow situation, whereas it is specified by the user in UnTRIMSediMorph. The suspended sediment transport is much larger (approx. 4 times) than bed load transport. During the storm period (7-9 February 2006), large suspended-transport rates are calculated by both UnTRIM and DELFT3D, but with slight differences in timing. Except for the stormy period, the UnTRIM and the DELFT3D results for the total sediment transport, as the sum of suspended and bed load transport, are approximately in the same range.

Considering the differences in model set-up (2-D/3-D), differences in the representation of sediment transport (transport formulas, sediment fractions and mode of transport), numerical factors (numerical diffusion/damping) and coupling hydrodynamics and waves, both models show quite comparable results. More detailed investigations are necessary to identify the influences of individual model parameterizations (grid topology/grid spacing, vertical resolution, sediment transport formulations) for a better explanation of differences in model results. However, in terms of a multimodel approach, the range of diverse model results seems quite important.

\section{Conclusions}

A morphodynamic modelling system for the German Bight has been set up using the DELFT3D and UnTRIMSediMorph modelling systems. Initial sensitivity studies illustrate the need for detailed sedimentological input data sets and improved estimates for specific sedimentological properties such as porosity, which strongly influence the model results. The approach to obtain a sedimentology, based on present-day hydrodynamic forcing, fails most evidently if geological structures from the past are present. The morphodynamic multi-model approach has been illustrated by sediment transport results for the German Bight. Even though results differ in detail, the large-scale spatial and temporal results are comparable. The proposed multi-model approach, as an extension to parameter variation of individual models, might be a way forward in order to assess model uncertainty in morphodynamic modelling following the path set by climate modellers.

Acknowledgements. The authors and the AufMod-team would like to thank the Federal Ministry of Education and Research (BMBF \#03KIS084) for funding of the research project.

\section{References}

BAW: Nordsee-Basismodell - Teil II: Modellsystem UnTRIMSediMorph-Unk, a) Hydrodynamik (UnTRIM-SediMorph), BAW-report A39550270116-2a, 2013.

Booij, N., Holthuijsen, L. H., and Ris, R. C.: The "SWAN" wave model for shallow water, Coast. Eng. Proc., 1, 25, 1996.

Casulli, V. and Zanolli, P.: Semi-Implicit Numerical Modelling of Non-Hydrostatic Free-surface Flows for Environmental Problems, Math. Comput. Model., 6, 1131-1149, 2002.

Figge, K.: Begleitheft zur Karte der Sedimentverteilung in der Deutschen Bucht 1:250000 (Nr. 2900) - DHI, 1981.

French, J. R. and Burningham, H.: Coastal geomorphology: trends and challenges, Prog. Phys. Geogr., 33, 117-129, 2009.

Kösters, F. and Winter, C.: Exploring German Bight coastal morphodynamics based on modelled bed shear stress, Geo Marine Lett., 34, 21-36, doi:10.1007/s00367-013-0346-y, 2014.

Lesser, G. R., Roelvink, J., van Kester, J., and Stelling, G.: Development and validation of a three-dimensional morphological model, Coastal Eng., 51, 883-915, 2004.

Lyard, F., Lefevre, F., Letellier, T., and Francis, O.: Modelling the global ocean tides: modern insights from FES2004, Ocean Dynam., 53, 394-415, 2006.

Malcherek, A., Piechotta, F., and Knoch, D.: Mathematical Module SediMorph. Technical report. Hamburg: Bundesanstalt für Wasserbau (BAW), 2005.

Plüß, A. and Heyer, H.: Morphodynamic Multi-Model Approach for the Elbe estuary. In Dohmen-Jansen and Hulscher, editors, River, Coastal and Estuarine Morphodynamics: RCEM 2007, 1, 113-117, London, Taylor and Francis Group, 2008.

Schneggenburger, C.: Spectral Wave Modelling with Nonlinear Dissipation, Hamburg: Dissertation, Universität Hamburg, FB Geowissenschaften, 1998.

Stelling, G. W.: On the construction of computational methods for shallow water flow problems, Rijkwaterstaat, the Netherlands: Technical Report 35, 1984.

Stelling, G. W.: Practical aspects of accurate tidal computations, J. Hydr. Eng., 112, 802-817, 1986.

Tebaldi, C. and Knutti, R.: The use of the multi-model ensemble in probabilistic climate projections, Philos. Trans. Roy. Soc. a Math. Phys. Eng. Sci., 365, 2053-2075, 2007.

Zeiler, M., Schwarzer, K., Bartholomä, A., and Ricklefs, K.: Seabed morphology and sediment dynamics, Die Küste, 74, 31-44, 2008. 